\title{
Solitary-wave solutions of the Degasperis-Procesi equation by means of the homotopy analysis method
}

\author{
S. Abbasbandy ${ }^{a, *, * *}$, E.J. Parkes ${ }^{b}$ \\ ${ }^{a}$ Department of Mathematics, Science and Research Branch, Islamic Azad \\ University, Tehran, 14778, Iran \\ ${ }^{\mathrm{b}}$ Department of Mathematics, University of Strathclyde, Glasgow G1 1XH, UK
}

\begin{abstract}
The homotopy analysis method is applied to the Degasperis-Procesi equation in order to find analytic approximations to the known exact solitary-wave solutions for the solitary peakon wave and the family of solitary smooth-hump waves. It is demonstrated that the approximate solutions agree well with the exact solutions. This provides further evidence that the homotopy analysis method is a powerful tool for finding excellent approximations to nonlinear solitary waves.
\end{abstract}

Key words: Degasperis-Procesi equation; Homotopy analysis method; Solitary-wave solution; Series solution

\section{Introduction}

The solution of nonlinear problems by analytic techniques is often rather difficult. Recently, the so-called homotopy analysis method (HAM) has been developed by Liao [1]. The HAM has been applied successfully to many nonlinear problems in engineering and science, such as applications in heat transfer [2], solving the generalized Hirota-Satsuma coupled KdV equation [3], in heat radiation [4], finding solitary-wave solutions for the fifth-order $\mathrm{KdV}$ equation [5], finding solitary wave solutions for the Kuramoto-Sivashinsky equation [6], finding the root of nonlinear

\footnotetext{
* Permanent Address: Department of Mathematics, Imam Khomeini International University, Ghazvin 34149-16818, Iran

** Corresponding author.

Email addresses: abbasbandy@yahoo.com (S. Abbasbandy),

ejp@maths.strath.ac.uk (E.J. Parkes).
} 
equations [7], boundary-layer flows over an impermeable stretched plate [8], unsteady boundary-layer flows over a stretching flat plate [9], exponentially decaying boundary layers [10], a nonlinear model of combined convective and radiative cooling of a spherical body [11], and many other problems (see [12-19], for example).

As discussed in [20], the family of equations

$$
u_{t}-u_{x x t}+(\gamma+1) u u_{x}=\gamma u_{x} u_{x x}+u u_{x x x}
$$

where $\gamma>1$ is a constant, contains only two integrable equations, namely the dispersionless Camassa-Holm ( $\mathrm{dCH}$ ) equation for which $\gamma=2$ and the Degasperis-Procesi (DP) equation for which $\gamma=3$. Explicit periodic and solitary travelling-wave solutions to the $\mathrm{dCH}$ and DP equations were found in [21] and [22], respectively. Weak travelling-wave solutions of the $\mathrm{CH}$ and $\mathrm{DP}$ equations, including exotic composite solutions, have been classified by Lenells [23,24]; these two papers are a useful source of references regarding properties of the $\mathrm{CH}$ and $\mathrm{DP}$ equations.

In [25] we found approximate analytic solutions for the family of solitary smoothhump waves of the $\mathrm{dCH}$ equation by using the HAM. The approximate analytic solution for the solitary peakon wave, which has a discontinuity at the crest, was found in [13] by using the HAM.

The aim of this paper is to apply the HAM to the DP equation in order to find analytic approximations to the solitary peakon wave and the family of solitary smooth-hump waves, all given in exact form by Vakhnenko and Parkes [22].

In Section 2 we give the exact solution for the solitary peakon, and present the exact solution for the family of solitary smooth-hump waves in an explicit and more convenient form than in [22]. In Sections 3 and 4 we formulate the HAM for finding approximate analytic solutions for the solitary peakon wave and the family of solitary smooth-hump waves, respectively. A brief conclusion is given in Section 5 .

\section{Exact solitary-wave solutions}

Vakhnenko and Parkes [22] looked for periodic and solitary-wave solutions of the DP equation

$$
u_{t}-u_{x x t}+4 u u_{x}=3 u_{x} u_{x x}+u u_{x x x}
$$

by seeking solutions in the form $u(x, t)=U(\eta)$, where $\eta:=x-c t-x_{0}$, and $c(>0)$ and $x_{0}$ are constants. They found three types of solitary wave characterized by a single parameter $A[22$, Section 2]. For $A=1$ there is a corner-wave solution known as a peakon. For $1<A<9 / 8$ there is a family of smooth-hump solitary waves.

In [22] the solitary-wave solutions were presented in terms of a new dependent 
variable $Z(\eta)$ related to $U(\eta)$ by

$$
U(\eta)=c[1+Z(\eta)]
$$

Here we summarize these solutions; the smooth-hump family is presented in an explicit and more convenient form than in [22].

\subsection{The peakon wave}

In $[22$, Section 2.3] the peakon is given by

$$
Z=e^{-|\eta|}-1
$$

so that

$$
U=c e^{-|\eta|} .
$$

The peakon is shown in [22, Fig. 2(c)].

\subsection{A family of smooth-hump solitary waves}

The family of smooth-hump solitary waves is given in [22, Section 2.4]. In parametric form, with $\tau$ as the parameter, $Z$ is given as an implicit function of $\eta$ by

$$
Z=\frac{z_{3}-z_{4} n \tanh ^{2} \tau}{1-n \tanh ^{2} \tau}, \quad \eta=\frac{\tau z_{2}}{s}+2 \tanh ^{-1}(\sqrt{n} \tanh \tau)
$$

where

$$
\begin{gathered}
n=\frac{z_{3}-z_{2}}{z_{4}-z_{2}}, \quad s=\frac{1}{2} \sqrt{\left(z_{4}-z_{2}\right)\left(z_{3}-z_{1}\right)}, \\
z_{1}=z_{2}=z_{L}<z_{3}<0<z_{4}, \quad z_{L}=b^{2}-1, \quad z_{3}=-b^{2}-b, \quad z_{4}=-b^{2}+b .
\end{gathered}
$$

The family-parameter is $b$ defined by

$$
b^{2}:=(1-\sqrt{9-8 A}) / 4 .
$$

With $1<A<9 / 8$ we have $0<b<1 / 2$. For each member of this family, $z_{L} \leq Z \leq$ $z_{3}, Z=z_{3}$ at the crest where $\tau=0$ (so that $\eta=0$ ), and $Z \rightarrow z_{L}$ as $|\tau| \rightarrow \infty$ (so that $|\eta| \rightarrow \infty)$. The member of this family with $b=1 / 4$ is shown in [22, Fig. 2(d)]. As $b \rightarrow 0$, the waves tend to the wave of maximum amplitude, namely the peakon (2.3).

Now, from (2.2) and (2.5)-(2.7), we obtain

$$
U=a W(\eta)+U_{\infty}
$$


where $a:=c\left(z_{3}-z_{L}\right)=c\left(1-b-2 b^{2}\right)$ is the amplitude, $U_{\infty}:=c\left(1+z_{L}\right)=c b^{2}$ and $W$ is given as an implicit function of $\eta$ by

$$
\begin{gathered}
W=\frac{\left(1+b-2 b^{2}\right)\left(1-\tanh ^{2} \tau\right)}{\left(1+b-2 b^{2}\right)-\left(1-b-2 b^{2}\right) \tanh ^{2} \tau}, \\
\eta=-2 \tau \sqrt{\frac{1-b^{2}}{1-4 b^{2}}}+2 \tanh ^{-1}\left(\sqrt{\frac{1-b-2 b^{2}}{1+b-2 b^{2}}} \tanh \tau\right) .
\end{gathered}
$$

$W$ is a solitary smooth-hump wave with unit amplitude. $W$ and its derivatives tend to zero as $|\eta| \rightarrow \infty, W(0)=1$ and $W^{\prime}(0)=0$.

\section{The HAM for the peakon solitary wave}

In this section we formulate the HAM in order to find from Eq. (2.1) an analytic approximation to the symmetric solitary peakon wave. It is convenient to introduce a new dependent variable $w(\eta)$ defined by

$$
u(x, t)=a w(\eta),
$$

where $a$ is the amplitude. Substitution of $u$ given by (3.1) into Eq. (2.1) gives (with $\left.x_{0}=0\right)$

$$
c\left(w^{\prime \prime \prime}-w^{\prime}\right)+a w\left(4 w^{\prime}-w^{\prime \prime \prime}\right)-3 a w^{\prime} w^{\prime \prime}=0 .
$$

Due to the assumed symmetry of the peakon, in the HAM we consider $w(\eta)$ only for $\eta \geq 0$. The first derivative at the crest of the peakon is not continuous. Hence the appropriate boundary conditions on $w$ for use in the HAM are

$$
w(0)=1, \quad w(+\infty)=0
$$

Our aim is to use the HAM to find analytic approximations to $a$ and $w(\eta)$. For simplicity, in the rest of this section we set $c=1$.

According to Eq. (3.2) and the boundary conditions (3.3), the solitary-wave solution can be expressed in the form

$$
w(\eta)=\sum_{m=1}^{+\infty} d_{m} e^{-m \eta}
$$

where the $d_{m}(m=1,2, \ldots)$ are coefficients to be determined. According to the rule of solution expression denoted by (3.4) and the boundary conditions (3.3), it is natural to choose

$$
w_{0}(\eta)=e^{-\eta}-\epsilon\left[e^{-2 \eta}-e^{-3 \eta}\right]
$$

as the initial approximation to $w(\eta)$, where $\epsilon$ is a parameter to be determined later. This choice follows the strategy adopted in [13] in the context of the peakon solution 
to the $\mathrm{CH}$ equation. We define an auxiliary linear operator $\mathcal{L}$ by

$$
\mathcal{L}[\phi(\eta ; p)]=\left(\frac{\partial^{3}}{\partial \eta^{3}}-3 \frac{\partial^{2}}{\partial \eta^{2}}+2 \frac{\partial}{\partial \eta}\right) \phi(\eta ; p)
$$

This has the property that

$$
\mathcal{L}\left[C_{1} e^{\eta}+C_{2} e^{2 \eta}+C_{3}\right]=0
$$

where $C_{1}, C_{2}$ and $C_{3}$ are constants. This choice of $\mathcal{L}$ is motivated by (3.4) and the later requirement that (3.16) should contain only zero constants, i.e. $C_{1}=C_{2}=$ $C_{3}=0$.

From (3.2) we define a nonlinear operator

$$
\mathcal{N}[\phi(\eta ; p), A(p)]:=\left(\frac{\partial^{3} \phi}{\partial \eta^{3}}-\frac{\partial \phi}{\partial \eta}\right)+A(p) \phi\left(4 \frac{\partial \phi}{\partial \eta}-\frac{\partial^{3} \phi}{\partial \eta^{3}}\right)-3 A(p) \frac{\partial \phi}{\partial \eta} \frac{\partial^{2} \phi}{\partial \eta^{2}}
$$

and then construct the homotopy

$$
\mathcal{H}[\phi(\eta ; p), A(p)]=(1-p) \mathcal{L}\left[\phi(\eta ; p)-w_{0}(\eta)\right]-\hbar p \mathcal{N}[\phi(\eta ; p), A(p)]
$$

where $\hbar$ is a nonzero auxiliary parameter. Setting $\mathcal{H}[\phi(\eta ; p), A(p)]=0$, we have the zero-order deformation equation

$$
(1-p) \mathcal{L}\left[\phi(\eta ; p)-w_{0}(\eta)\right]=\hbar p \mathcal{N}[\phi(\eta ; p), A(p)]
$$

subject to the boundary conditions

$$
\phi(0 ; p)=1, \quad \phi(+\infty ; p)=0,
$$

where $p \in[0,1]$ is an embedding parameter. When the parameter $p$ increases from 0 to 1 , the solution $\phi(\eta ; p)$ varies from $w_{0}(\eta)$ to $w(\eta)$, and $A(p)$ varies from $a_{0}$ to $a$, where $a_{0}$ is the initial value of the wave amplitude. If this continuous variation is smooth enough, the Maclaurin's series with respect to $p$ can be constructed for $\phi(\eta ; p)$ and $A(p)$, and further, if these two series are convergent at $p=1$, we have

$$
w(\eta)=w_{0}(\eta)+\sum_{m=1}^{+\infty} w_{m}(\eta), \quad a=a_{0}+\sum_{m=1}^{+\infty} a_{m}
$$

where

$$
w_{m}(\eta)=\left.\frac{1}{m !} \frac{\partial^{m} \phi(\eta ; p)}{\partial p^{m}}\right|_{p=0}, \quad a_{m}=\left.\frac{1}{m !} \frac{\partial^{m} A(p)}{\partial p^{m}}\right|_{p=0}
$$

For brevity, we define the vectors

$$
\vec{w}_{k}=\left\{w_{0}, w_{1}, \ldots, w_{k}\right\}, \quad \vec{a}_{k}=\left\{a_{0}, a_{1}, \ldots, a_{k}\right\} .
$$


Differentiating Eqs. (3.10) and (3.11) $m$ times with respect to $p$, then setting $p=0$, and finally dividing by $m$ !, we obtain the $m$ th-order deformation equation

$$
\mathcal{L}\left[w_{m}(\eta)-\chi_{m} w_{m-1}(\eta)\right]=\hbar R_{m}\left(\vec{w}_{m-1}, \vec{a}_{m-1}\right), \quad(m=1,2,3, \ldots)
$$

subject to the boundary conditions

$$
w_{m}(0)=0, \quad w_{m}(\infty)=0,
$$

where

$$
R_{m}=w_{m-1}^{\prime \prime \prime}-w_{m-1}^{\prime}+\sum_{n=0}^{m-1} \sum_{i=0}^{n} a_{i}\left(w_{n-i}\left[4 w_{m-n-1}^{\prime}-w_{m-n-1}^{\prime \prime \prime}\right]-3 w_{n-i}^{\prime} w_{m-n-1}^{\prime \prime}\right),
$$

and

$$
\chi_{m}=\left\{\begin{array}{l}
0, m \leq 1 \\
1, m>1
\end{array}\right.
$$

The general solution of Eq. (3.14) is

$$
w_{m}(\eta)=\hat{w}_{m}(\eta)+C_{1} e^{\eta}+C_{2} e^{2 \eta}+C_{3},
$$

where $C_{1}, C_{2}$ and $C_{3}$ are constants and $\hat{w}_{m}(\eta)$ is the particular solution of Eq. (3.14) that contains the unknown term $a_{m-1}$. According to the boundary condition (3.15) at infinity and the rule of solution expression (3.4), the constants $C_{1}, C_{2}$ and $C_{3}$ must be zero. Due to the boundary condition (3.15) at $\eta=0$, the unknown $a_{m-1}$ is determined by the linear algebraic equation

$$
\hat{w}_{m}(0)=0 \text {. }
$$

In this way, we derive $w_{m}(\eta)$ and $a_{m-1}$ for $m=1,2,3, \ldots$, successively. At the $M$ th-order approximation, we have the analytic solution of Eq. (3.2), namely

$$
w(\eta) \approx W_{M}(\eta)=\sum_{m=0}^{M} w_{m}(\eta), \quad a \approx A_{M}=\sum_{m=0}^{M} a_{m} .
$$

The auxiliary parameter $\hbar$ can be employed to adjust the convergence region of the series (3.18) in the homotopy analysis solution. By means of the so-called $\hbar-$ curve, it is straightforward to choose an appropriate range for $\hbar$ which ensures the convergence of the solution series. As pointed out by Liao [1], the appropriate region for $\hbar$ is a horizontal line segment.

Our solution series contain the auxiliary parameters $\hbar$ and $\epsilon$. We can choose appropriate values of $\hbar$ and $\epsilon$ to ensure that the two solution series converge. For a given $\hbar$, we can investigate the influence of $\epsilon$ on the convergence of $a$ by plotting the curve of $a$ versus $\epsilon$, as shown in Fig. 1. We find that the appropriate region for $\epsilon$ is $-1<\epsilon<1$. In the same way, we can plot $\hbar$-curves for $a$ with any given $\epsilon$, as 
shown in Fig. 2. Clearly, $a \approx 1$ which agrees with the expected value $c=1$. It now follows that, for convergent solution series, we can choose $\hbar=-3$ and $\epsilon=-0.5$, for example. The corresponding 20th-order approximation for $w(\eta)$ agrees well with the exact solution, i.e. $w=e^{-|\eta|}$, as shown in Fig. 3 .

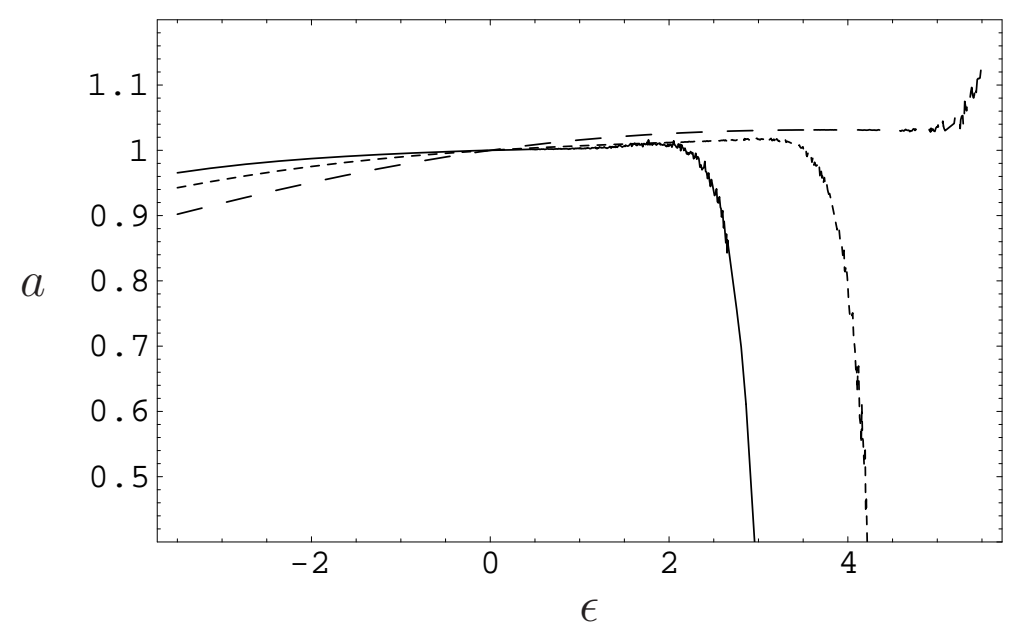

Fig. 1: The curves of the wave amplitude $a$ versus $\epsilon$ for the 10th-order approximation. Solid curve: $\hbar=-4$; dotted curve: $\hbar=-3$; dashed curve: $\hbar=-2$.

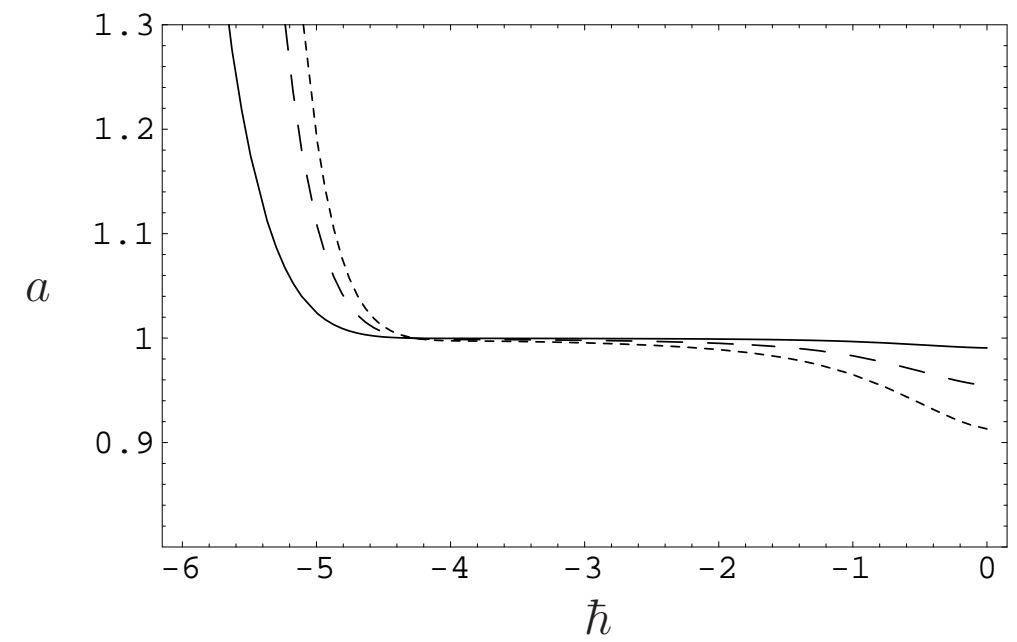

Fig. 2: The curves of the wave amplitude $a$ versus $\hbar$ for the 15 th-order approximation. Solid curve: $\epsilon=-0.5$; dotted curve: $\epsilon=-1.5$; dashed curve:

$$
\epsilon=-1 \text {. }
$$

\section{The HAM for the family of smooth-hump solitary waves}

In this section we formulate the HAM in order to find from Eq. (2.1) an analytic approximation to the family of symmetric solitary smooth-hump waves, with familyparameter $U_{\infty}$, given exactly in Section 2.2. 
It is convenient to introduce a new dependent variable $w(\eta)$ defined by

$$
u(x, t)=U(\eta):=a w(\eta)+U_{\infty}, \quad 0<U_{\infty}<c / 4
$$

where $a$ is the amplitude, $u \rightarrow U_{\infty}$ as $|\eta| \rightarrow \infty, U_{\infty}:=c b^{2}$ with $0<b<1 / 2$, and $w(\eta)$ is a solitary smooth-hump wave of unit amplitude such that $w(0)=1$, $w^{\prime}(0)=0, w \rightarrow 0$ as $|\eta| \rightarrow \infty$, and $w(\eta)=w(-\eta)$. Substitution of $u$ given by (4.1) into Eq. (2.1) gives

$$
c\left(w^{\prime \prime \prime}-w^{\prime}\right)+\left(a w+b^{2} c\right)\left(4 w^{\prime}-w^{\prime \prime \prime}\right)-3 a w^{\prime} w^{\prime \prime}=0 .
$$

Due to the assumed symmetry of the solitary waves, in the HAM we consider $w(\eta)$ only for $\eta \geq 0$. Hence the appropriate boundary conditions on $w$ for use in the HAM are

$$
w(0)=1, \quad w^{\prime}(0)=0, \quad w(+\infty)=0 .
$$

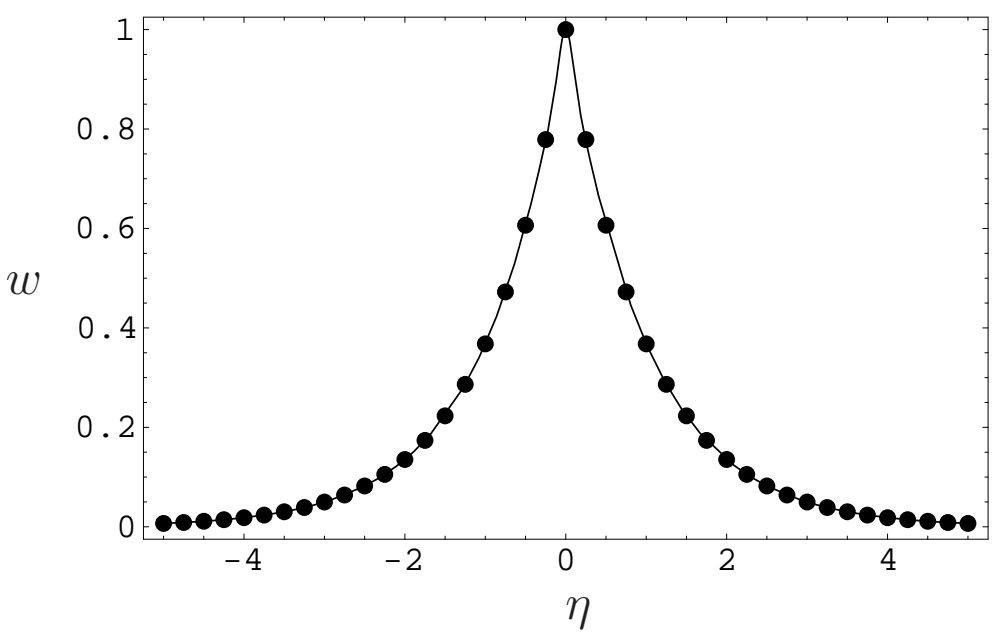

Fig. 3: The analytic approximation for $w(\eta)$ when $\hbar=-3$ with $\epsilon=-0.5$ and the exact solution $w(\eta)=-e^{|\eta|}$. Solid curve: 20th-order approximation; symbols: exact solution.

Our aim is to use the HAM to find analytic approximations to $a$ and $w(\eta)$. We will demonstrate that these approximations are in good agreement with the exact expressions $c\left(1-b-2 b^{2}\right)$ and $W(\eta)$, respectively, as derived in Section 2.2.

First we write

$$
w(\eta) \approx B \exp (-\mu \eta), \text { as } \eta \rightarrow \infty,
$$

where $\mu>0$ and $B$ are constants. Substituting (4.4) into (4.2) and balancing the main terms, we have

$$
\mu^{2}=\frac{1-4 b^{2}}{1-b^{2}} .
$$

Now we put $\xi=\mu \eta$ so that Eq. (4.2) becomes

$$
c\left(1-4 b^{2}\right)\left(w^{\prime \prime \prime}-w^{\prime}\right)+a w\left(4 w^{\prime}-\mu^{2} w^{\prime \prime \prime}\right)-3 \mu^{2} a w^{\prime} w^{\prime \prime}=0,
$$


where the prime denotes the derivative with respect to $\xi$. For simplicity, in the rest of this section we set $c=1$.

According to Eq. (4.5) and the boundary conditions (4.3), the solitary-wave solution can be expressed in the form

$$
w(\xi)=\sum_{m=1}^{+\infty} d_{m} e^{-m \xi}
$$

where the $d_{m}(m=1,2, \ldots)$ are coefficients to be determined.

We define an auxiliary linear operator $\mathcal{L}$ by

$$
\mathcal{L}[\phi(\xi ; p)]=\left(1-4 b^{2}\right)\left(\frac{\partial^{3}}{\partial \xi^{3}}-\frac{\partial}{\partial \xi}\right) \phi(\xi ; p),
$$

with the property

$$
\mathcal{L}\left[C_{1} e^{-\xi}+C_{2} e^{\xi}+C_{3}\right]=0,
$$

where $C_{1}, C_{2}$ and $C_{3}$ are constants. This choice of $\mathcal{L}$ is motivated by (4.6) and the later requirement that (4.14) should contain only one non-zero constant, namely $C_{1}$.

In this case, the nonlinear operator $\mathcal{N}[\phi(\xi ; p)]$ is defined as

$$
\begin{gathered}
\mathcal{N}[\phi(\xi ; p), A(p)]:=\left(1-4 b^{2}\right)\left(\frac{\partial^{3} \phi}{\partial \xi^{3}}-\frac{\partial \phi}{\partial \xi}\right)+A(p) \phi\left(4 \frac{\partial \phi}{\partial \xi}-\mu^{2} \frac{\partial^{3} \phi}{\partial \xi^{3}}\right) \\
-3 \mu^{2} A(p) \frac{\partial \phi}{\partial \xi} \frac{\partial^{2} \phi}{\partial \xi^{2}}
\end{gathered}
$$

and the homotopy $\mathcal{H}$ is defined as in (3.9). Also, the zero-order deformation equation is defined as

$$
(1-p) \mathcal{L}\left[\phi(\xi ; p)-w_{0}(\xi)\right]=\hbar p \mathcal{N}[\phi(\xi ; p), A(p)],
$$

subject to the boundary conditions

$$
\phi(0 ; p)=1,\left.\quad \frac{\partial \phi(\xi ; p)}{\partial \xi}\right|_{\xi=0}=0, \quad \phi(+\infty ; p)=0
$$

where $p \in[0,1]$ is an embedding parameter and $w_{0}(\xi)=2 e^{-\xi}-e^{-2 \xi}$. Differentiating Eqs. (4.10) and (4.11) $m$ times with respect to $p$, then setting $p=0$, and finally dividing by $m$ !, we obtain the $m$ th-order deformation equation

$$
\mathcal{L}\left[w_{m}(\xi)-\chi_{m} w_{m-1}(\xi)\right]=\hbar R_{m}\left(\vec{w}_{m-1}\right), \quad(m=1,2,3, \ldots)
$$

subject to the boundary conditions

$$
w_{m}(0)=0, \quad w_{m}^{\prime}(0)=0, \quad w_{m}(\infty)=0,
$$


where $R_{m}$ is defined as

$$
\begin{gathered}
R_{m}=\left(1-4 b^{2}\right)\left(w_{m-1}^{\prime \prime \prime}-w_{m-1}^{\prime}\right) \\
+\sum_{n=0}^{m-1} \sum_{i=0}^{n} a_{i}\left(w_{n-i}\left[4 w_{m-n-1}^{\prime}-\mu^{2} w_{m-n-1}^{\prime \prime \prime}\right]-3 \mu^{2} w_{n-i}^{\prime} w_{m-n-1}^{\prime \prime}\right) .
\end{gathered}
$$

The general solution of Eq. (4.12) is

$$
w_{m}(\xi)=\hat{w}_{m}(\xi)+C_{1} e^{-\xi}+C_{2} e^{\xi}+C_{3}
$$

where $C_{1}, C_{2}$ and $C_{3}$ are constants and $\hat{w}_{m}(\xi)$ is a particular solution of Eq. (4.12). Using (4.6), we have $C_{2}=C_{3}=0$. The unknowns $C_{1}$ and $a_{m-1}$ are governed by

$$
\hat{w}_{m}(0)+C_{1}=0, \quad \hat{w}_{m}^{\prime}(0)-C_{1}=0 .
$$

Thus, the unknown $a_{m-1}$ is obtained by solving the linear algebraic equation

$$
\hat{w}_{m}(0)+\hat{w}_{m}^{\prime}(0)=0,
$$

and thereafter $C_{1}$ is given by

$$
C_{1}=-\hat{w}_{m}(0)
$$

To ensure of convergence of the HAM, we first focus on how to choose an appropriate value of $\hbar$. We can investigate the influence of $\hbar$ on the series of $a$ by means of the $\hbar$-curve. The appropriate region for $\hbar$ in this case is $-3.1<\hbar<-1.2$, as shown in Fig. 4 for $b=\frac{1}{4}$. In this case the exact value of $a=c\left(1-b-2 b^{2}\right)$ is 0.625 . In general, as long as the series of amplitude is convergent, the corresponding series for $w(\xi)$ is also convergent. For example, when $\hbar=-2$, our analytic solution converges. This is demonstrated in Fig. 5 where it can be seen that the 10th-order approximation for $w$ as a function of $\eta(\equiv \xi / \mu)$ agrees well with the exact solution given by Eqs. (2.10) and (2.11).

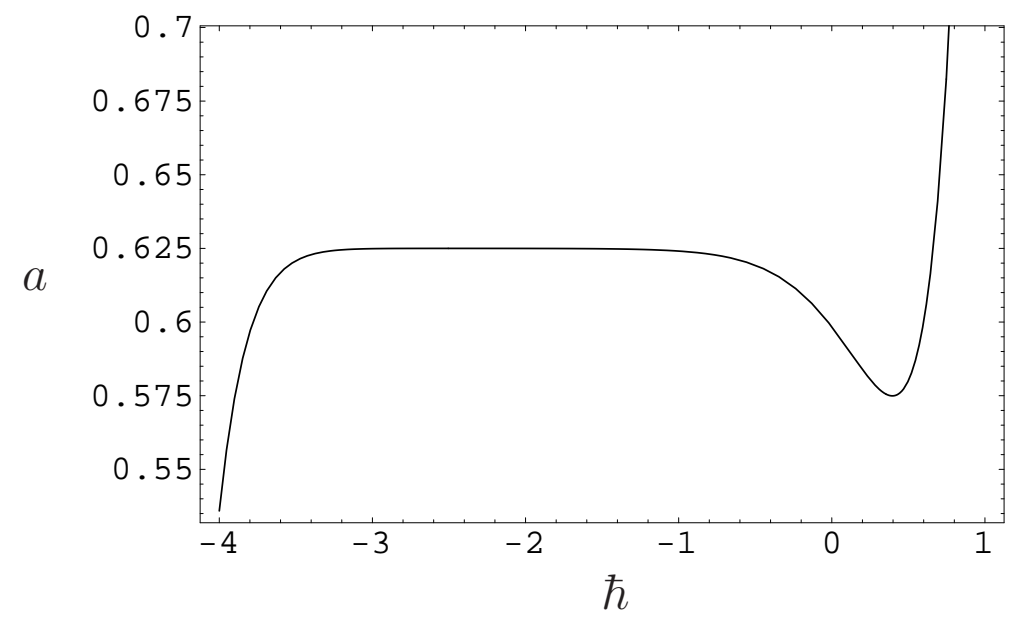

Fig. 4: The $\hbar$-curve for the wave amplitude $a$ at the 15 th-order approximation with $b=\frac{1}{4}$. 
The value of the amplitude is shown in Table 1 . The so-called homotopy-Padé technique (see [1]) is employed, which greatly accelerates the convergence. Clearly, the amplitude converges to the exact value 0.625 .

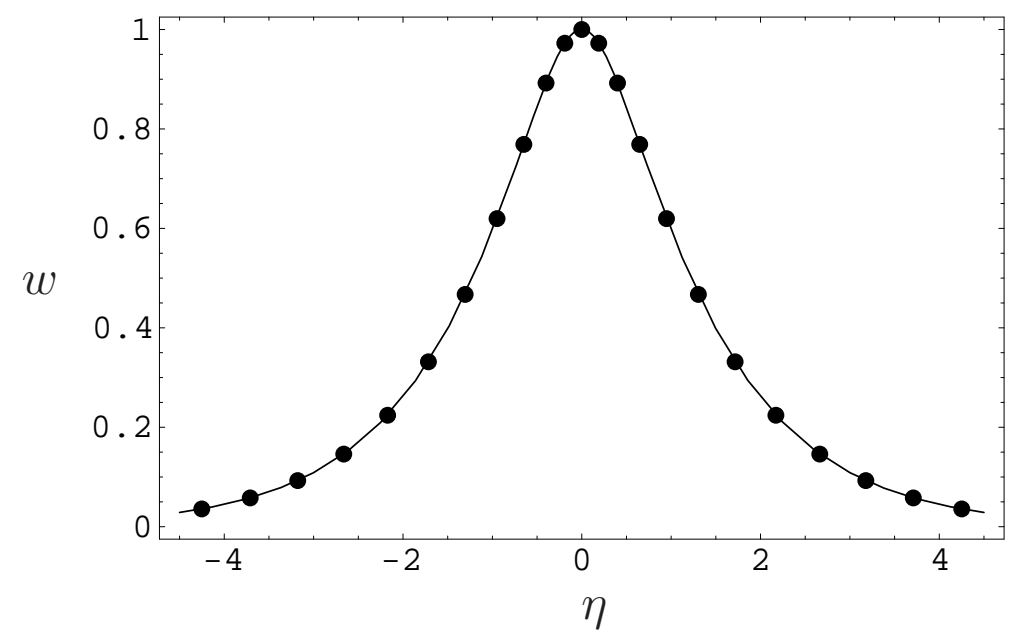

Fig. 5: The analytic approximation for $w$ when $\hbar=-2$ with $b=\frac{1}{4}$ and the exact solution given by Eqs. (2.10) and (2.11). Solid curve: the 10th-order approximation; symbols: exact solution.

Table 1: Results for $[m, m]$ Homotopy-Padé approach

\begin{tabular}{c|cc}
\hline Order of approximation & {$[m, m]$} & $a$ \\
\hline 2 & {$[1,1]$} & 0.644857 \\
4 & {$[2,2]$} & 0.624729 \\
6 & {$[3,3]$} & 0.625302 \\
8 & {$[4,4]$} & 0.624991 \\
10 & {$[5,5]$} & 0.625132 \\
12 & {$[6,6]$} & 0.625 \\
14 & {$[7,7]$} & 0.625
\end{tabular}

\section{Conclusions}

We have applied the homotopy analysis method (HAM) to the Degasperis-Procesi equation (2.1) to obtain analytic approximations to known solitary-wave solutions as given in [22]. In Sections 3 and 4, the methods used are very similar to the ones used for the dCH equation in [13] and [25], respectively. For the peakon solution in Section 3, and the family of smooth-hump waves in Section 4, the amplitude of the solitary waves was treated as an unknown to be determined by the HAM. In all the cases considered, the HAM gave excellent agreement with the known solutions. 
In [22] it was shown that the DP equation has a solitary loop-like solution. In [26] we attempted to formulate the HAM in order to find an analytic approximation to this exact solution. The formulation involved the introduction of a new independent variable as was done in [27] for the short-pulse equation; the resulting equation, corresponding to (4.2), involved higher-order nonlinearities. We found that the approximate solution did not agree well with the exact solution. Resolution of this problem is ongoing.

The HAM provides us with a convenient way to control the convergence of approximation series; this is a fundamental qualitative difference between the HAM and other methods for finding approximate solutions. The examples in this paper give further confirmation of the power of the HAM to solve complicated nonlinear problems.

Acknowledgements. The authors would like to thank anonymous referees for valuable suggestions.

\section{References}

[1] Liao SJ. Beyond perturbation: introduction to the homotopy analysis method. Boca Raton: Chapman \& Hall/CRC Press; 2003.

[2] Abbasbandy S. The application of homotopy analysis method to nonlinear equations arising in heat transfer. Phys Lett A 2006;360:109-13.

[3] Abbasbandy S. The application of homotopy analysis method to solve a generalized Hirota-Satsuma coupled KdV equation. Phys Lett A 2007;361:478-83.

[4] Abbasbandy S. Homotopy analysis method for heat radiation equations. Int Commun Heat Mass Transf 2007;34:380-7.

[5] Abbasbandy S, Samadian Zakaria F. Soliton solutions for the fifth-order KdV equation with the homotopy analysis method. Nonlinear Dynam 2008;51:83-87.

[6] Abbasbandy S. Solitary wave solutions to the Kuramoto-Sivashinsky equation by means of the homotopy analysis method. Nonlinear Dynam 2008;52:35-40.

[7] Abbasbandy S, Tan Y, Liao SJ. Newton-Homotopy analysis method for nonlinear equations. Appl Math Comput 2007;188:1794-1800.

[8] Liao SJ. A new branch of solutions of boundary-layer flows over an impermeable stretched plate. Int J Heat Mass Transfer 2005;48:2529-39.

[9] Liao SJ. Series solutions of unsteady boundary-layer flows over a stretching flat plate. Stud Appl Math 2006;117:239-64.

[10] Liao SJ, Magyari E. Exponentially decaying boundary layers as limiting cases of families of algebraically decaying ones. Z Angew Math Phys (ZAMP) 2006;57:77792. 
[11] Liao SJ, Su J, Chwang AT. Series solutions for a nonlinear model of combined convective and radiative cooling of a spherical body. Int $\mathrm{J}$ Heat Mass Transfer 2006;49:2437-45.

[12] Tan Y, Xu H, Liao SJ. Explicit series solution of travelling waves with a front of Fisher equation. Chaos, Solitons \& Fractals 2007;31:462-72.

[13] Wu W, Liao SJ. Solving solitary waves with discontinuity by means of the homotopy analysis method. Chaos, Solitons \& Fractals 2005;26:177-85.

[14] Hayat T, Khan M. Homotopy solutions for a generalized second-grade fluid past a porous plate. Nonlinear Dynam 2005;42:395-405.

[15] Hayat T, Khan M, Ayub M. On non-linear flows with slip boundary condition. Z Angew Math Phys (ZAMP) 2005;56:1012-29.

[16] Liao SJ, Cheung K. Homotopy analysis of nonlinear progressive waves in deep water. J Eng Math 2003;45:105-16.

[17] Sajid M, Hayat T, Asghar S. On the analytic solution of the steady flow of a fourth grade fluid. Phys Lett A 2006;355:18-26.

[18] Tan Y, Abbasbandy S. Homotopy analysis method for quadratic Riccati differential equation. Commun Nonlinear Sci Numer Simul 2008;13:539-546.

[19] Wang C. Analytic solutions for a liquid film on an unsteady stretching surface. Heat Mass Transfer 2006;42:759-66.

[20] Degasperis A, Holm D, Hone A. A new integrable equation with peakon solutions. Theor Math Phys 2002;133:1461-72.

[21] Parkes EJ, Vakhnenko VO. Explicit solutions of the Camassa-Holm equation. Chaos, Solitons \& Fractals 2005;26:1309-16.

[22] Vakhnenko VO, Parkes EJ. Periodic and solitary-wave solutions of the DegasperisProcesi equation. Chaos, Solitons \& Fractals 2004;20:1059-73.

[23] Lenells J. Traveling wave solutions of the Camassa-Holm equation. J Diff Eq $2005 ; 217: 393-430$.

[24] Lenells J. Traveling wave solutions of the Degasperis-Procesi equation. J Math Anal Appl 2005;306:72-82.

[25] Abbasbandy S, Parkes EJ. Solitary smooth-hump solutions of the Camassa-Holm equation by means of the homotopy analysis method. Chaos, Solitons \& Fractals 2008;36:581-591.

[26] Abbasbandy S, Parkes EJ. The family of inverted-loop solitary wave solutions of the Degasperis-Procesi equation by means of the homotopy analysis method. International Conference on 'Recent Developments in Fluid Mechanics', Islamabad, Pakistan, 2007.

[27] Parkes EJ, Abbasbandy S. Finding the one-loop soliton solution of the short-pulse equation by means of the homotopy analysis method. Numer. Methods Partial Differential Equations, DOI: 10.1002/num.20348. 